\title{
Dynamical Measurements in Vehicles \\ by Transputer Technology
}

\author{
W. EISMANN* and W. SCHIEHLEN*
}

\begin{abstract}
SUMMARY
The experimental validation of mechanical models in vehicle dynamics requires dynamical measurements while driving. Parallel processing using transputer technology allows long time measurements at high sampling rate without any loss of data as well as online computation resulting in a very efficient tool forvibration monitoring and parameteridentification of vehiclesystems. The paper presents the realization of a mobile data acquisition system based on transputer technology and some applications of the system to vibration monitoring and identification of a rolling unbalanced vehicle wheel. The measurements are performed with a standard passenger car of a German trademark.
\end{abstract}

\section{INTRODUCTION}

During the last decade the technique of measurement and data acquisition in vehicles while driving was improved essentially. Experimental cars were equipped with realtime computer systems, Hees, et al. [2]. Vehicle design, testing and validation of their mechanical models used to be the main motivation, Zomotor [7]. However, more and more measurements and real time evaluations are applied for monitoring vehicles, too, to enhance driving safty, comfort and performance.

As an example of how recent measurement hardware in combination with symbolically modeled multibody systems may fullfill complex tasks in vibration monitoring of vehicles, this paper presents the vibration monitoring and the identification of vehicle system parameters based on acceleration measurements while driving with unbalanced wheels.

A main difficulty with the task described is the stochastic excitation by the rough road surface which cannot be measured directly. Hence, the covariance method, Kallenbach [5], may be applied to identify unknown covariances due to the road excitation together with the system parameters as reported in Eismann, Schiehlen [4].

On the other hand, considering the stochastic road excitation as measuring noise one gets well conditioned deterministic identification relations due to the excitation by unbalanced wheels, which are found analytically as functions of the driving speed, Eismann [3]. This kind of identification is based on crosscorrelation between the measured signals and harmonic model functions generated from the measured wheel frequency. The resulting amplitudes and phase angles represent samples of the system frequency response function with respect to un-

- Institute B of Mechanics, University of Stuttgart,Pfaffenwaldring 9,D-70550Stuttgart, Germany. 
balancy excitation. The technique works with all kind of wheeled vehicles on roads and railway tracks.

For the demands of this algorithm the mobile data acquisition system has to process wheel impulse transducer signals, to compute the harmonic model functions and to sample mechanical quantities to be measured, in this case accelerations.

\section{MOBILE DATA ACQUISITION}

The mobile data acquisition system provided with the Institute B of Mechanics, University of Stuttgart, consists of a portable frontend based on transputer technology, a laptop PC as host computer and the sensors, i. e., acceleration sensors and impulse transducers, see Fig. 1 . The system might be power supplied either by $12 \mathrm{~V} \mathrm{DC}$ on the vehicle or by $220 \mathrm{~V} \mathrm{AC}$ at the laboratory. It is possible to operate the system without running a hard disk, buffering the sampled data to the 32 MByte chip memory the frontend is equipped with. Hence, the data sampled while driving might be transferred to the hard disk at a later stop.

During operation there are two main parallel processes. Firstly the user interface process running on the PC, and secondly the data acquisition process running on the transputer system.

The user interface process on the PC enables configuration and control of the whole system and graphical visualization of data. Configuration means activation of input channels, scaling of data to physical units, definition of cutoff frequency and amplification of the analog filters as well as definition of sampling rate and FFT-parameters like block length, kind of windowing, kind of averaging and, of course, marking the two FFT-channels.

Graphical online visualization is performed with sampled data as well as with computed values. The presentation might be stopped at any time to zoom in or to display the coordinates of single points numerically. Further, old data files might be replayed to the memory board of the transputer system, too, to be visualized again or to recompute the FFT of any desired time intervall.

Docking the mobile data acquisition system to the stationary computer network at the laboratory is enabled by an Ethernet adaptor linked with the mobile PC.

The data acquisition process on the transputer system itself consists of four subprocesses running simultaneously:

i) controlling the Analog-Digital-Converter board,

ii) managing the impuls transducer board, i. e., computing actual angular locations from impuls transducer counts, computing the harmonic model functions and controlling the Digital-Analog-Converter and sending angular location and angular velocity values to the memory board,

iii) performing the online data processing, i. e., computing Fast-FourierTransforms (FFT) of two user defined analog channels and postprocessing to get spectral density functions, coherence and transfer function,

iv) serving the $\mathrm{PC}$-interface allowing user interactions like changing the cutoff frequency of the analog filters. 


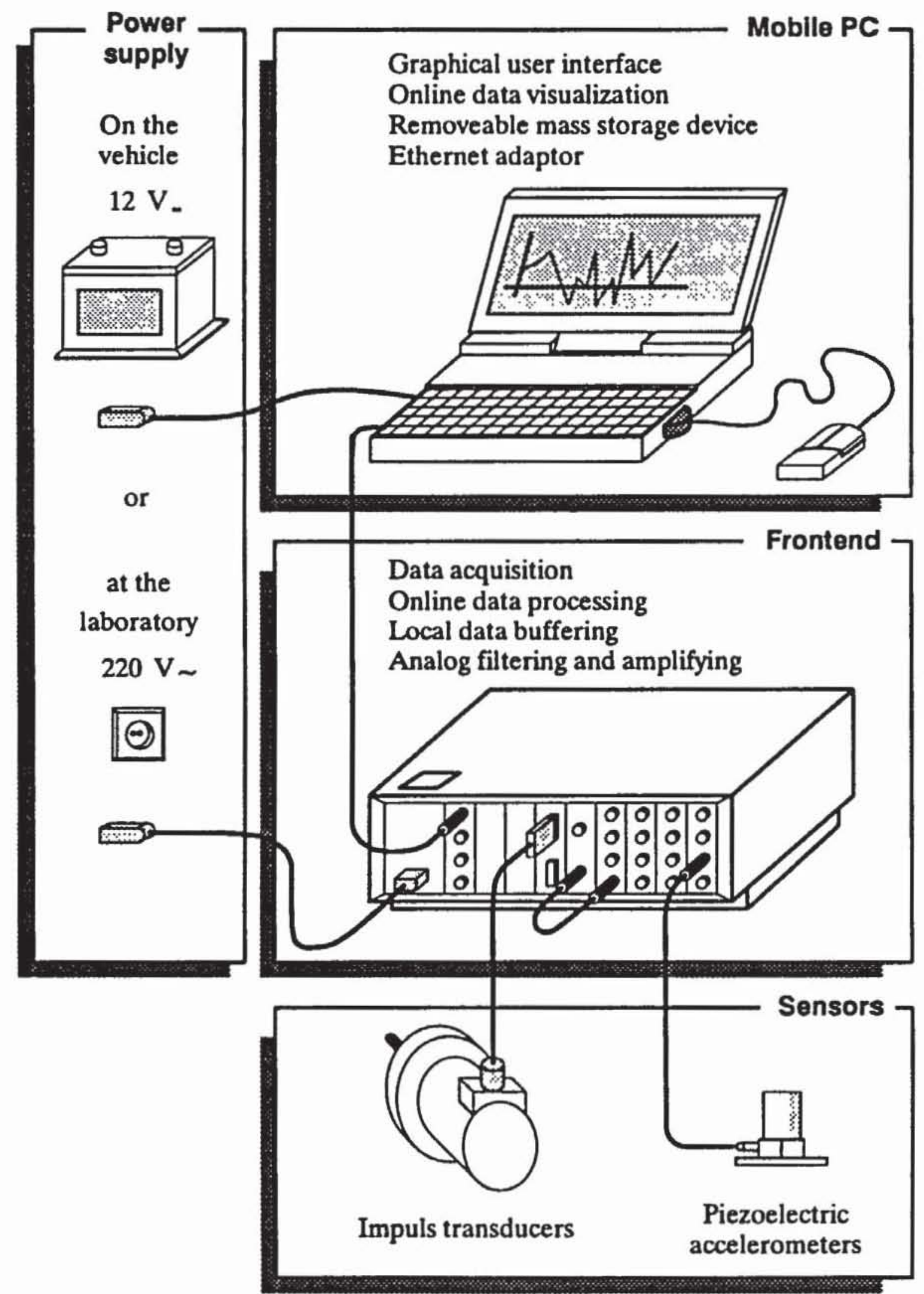

Fig. 1: Configuration of the mobile data acquisition system 118 
The topology of the transputer system and the flows of information and data are shown in more detail in Fig. 2. After loading and starting the transputer process the central node TPM-I/O equipped with a transputer T 800 further controls the frontend. Information like sampling rate or cutoff frequency is received from the host and is sent to the impuls transducer interface TS 2, to the analog filter boards SCF 4 and/or to the 12-bit-AD-converter TPM-ADC 1, respectively. Data reaching at the TS 2 board are collected by the wheel impuls transducers. This data will be processed locally by a transputer T222.

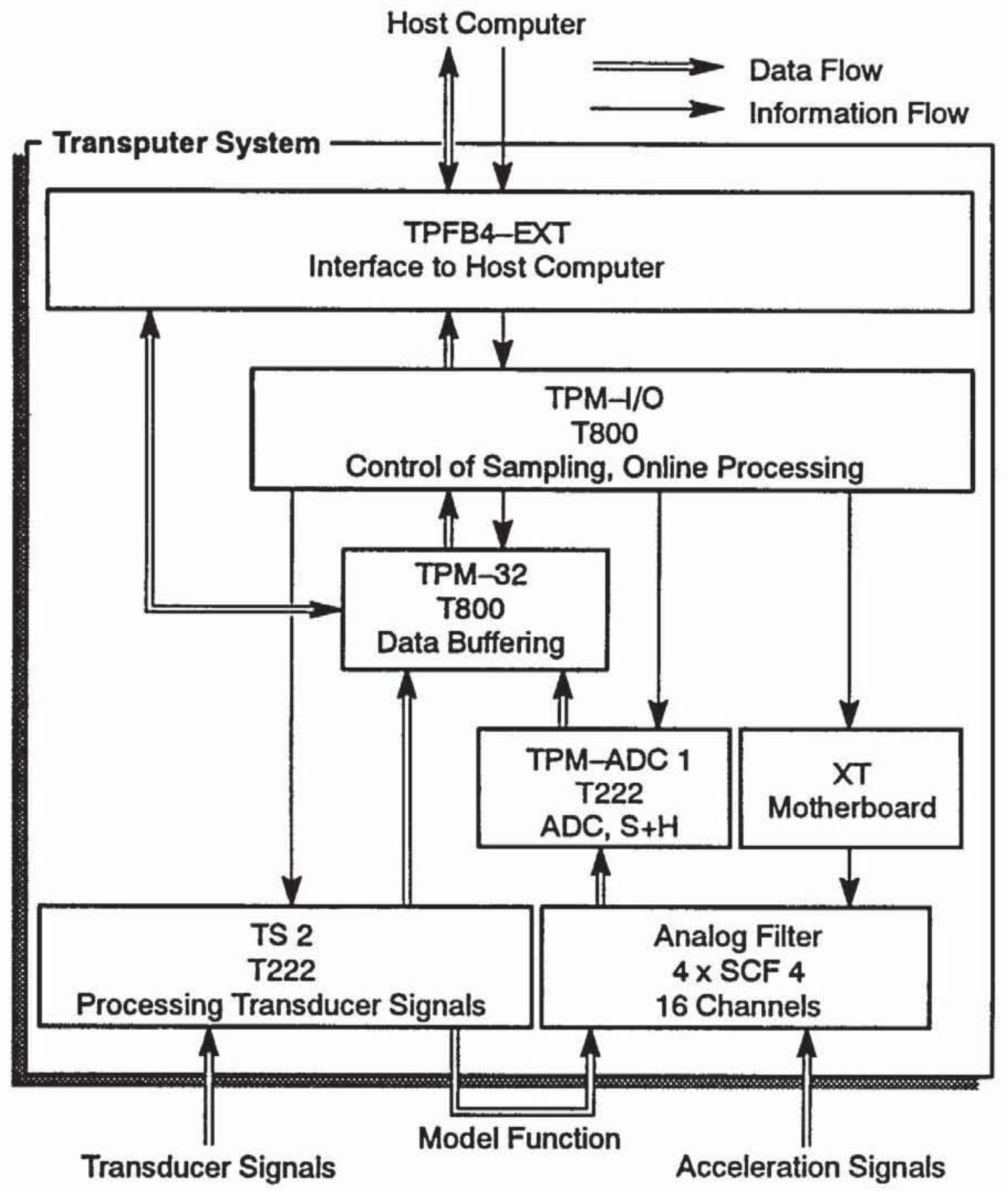

Fig. 2: Topology of the fontend based on transputer technology 
The output is the actual angular location of the wheels, the actual angular velocity and the actual values of the harmonic model functions. Angular location and angular velocity are directly transferred to the memory board TPM 32 with respect to the trigger signal provided by the TPM-I/O board. The digital values of harmonic model functions are converted analog ones and are resampled as additional analog channels to ensure vanishing relative phase shift between the acceleration signals and the model functions with respect to analog filtering.

The AD-converter board TMP-ADC 1 is equipped with sample and hold circuits to perform optimal synchronization due to multiplexed sampling of the 16 input channels. The board is locally controlled by a T222 transputer. The digital data are put to the memory board TPM -32 and, in addition, data blocks might be transferred to the central node to perform online processing. The data blocks and the results of online processing are delivered to the graphical interface on the PC.

After sampling was stopped, data buffered on the memory board of the frontend might be transferred to the hard disk of the PC, too. It should be mentioned that the frontend is equipped with recargeable batteries to keep the system running for at least 2 minutes in case of loss of power supply. The maximal sampling rate is about $3 \mathrm{kHz}$.

Fig. 3 shows the experimental car equipped with the transputer frontend and the laptop PC.

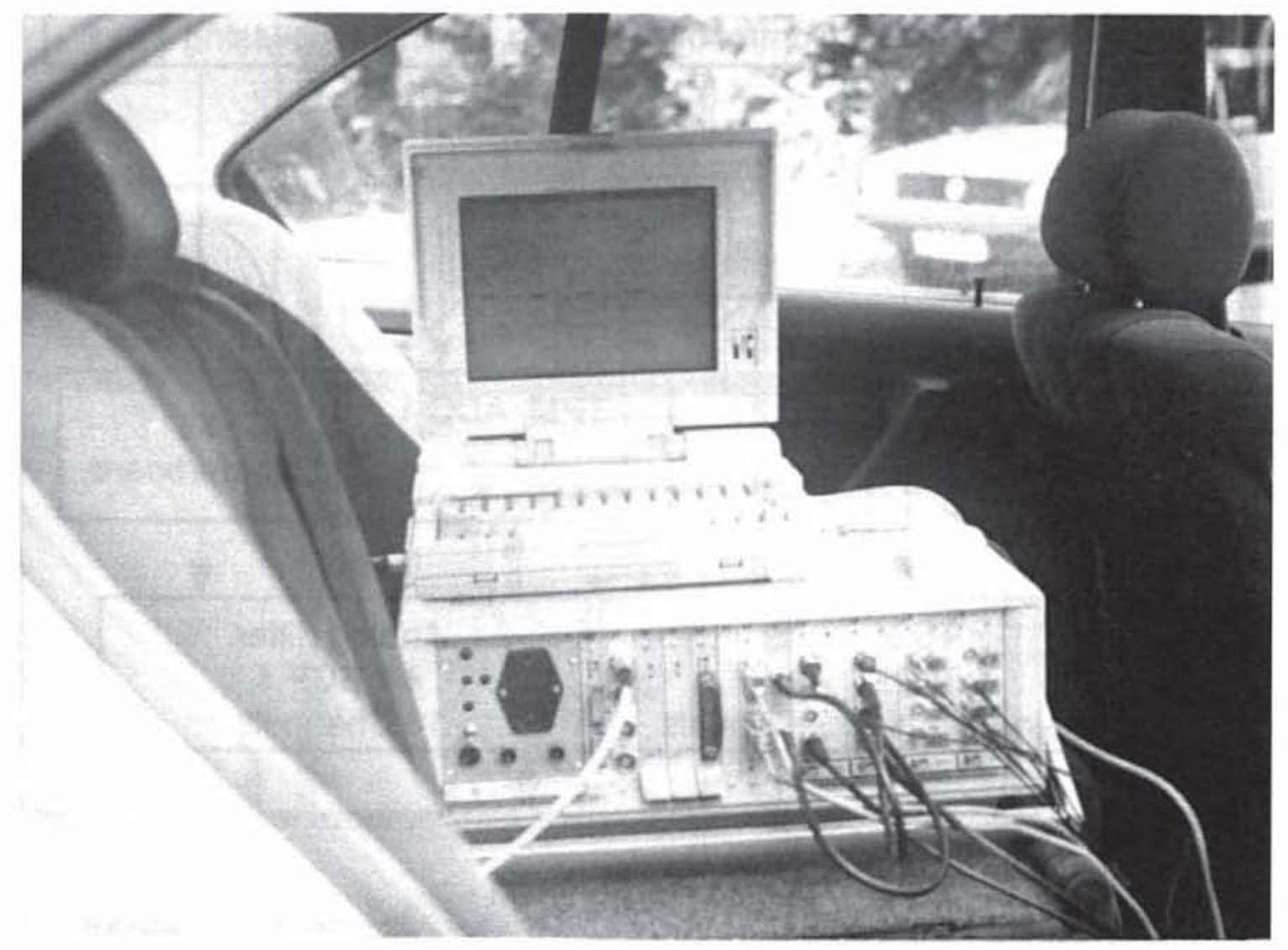

Fig. 3:

Experimental vehicle equipped with the mobile data acquisition system 


\section{VIBRATION MONITORING}

As a first example for the capability of the presented technology spatial vibration monitoring is considered. A mechanical model for vibration monitoring has to accommodate the real motion of the vehicle system. For the identification of static and dynamic wheel unbalances, for instance, based on measurements at the wheel support, the real spatial vibrations of the wheel support with respect to any given unbalancy excitation has to be observed.

The spatial vibration of a rigid body about a given equilibrium position is definitely determined by the twist $\left(\omega, \mathbf{v}_{\mathrm{c}}\right)$ of the center of gravity. If only translational acceleration sensors are used, direct measuring of the twist is not possible. Then, it has to be computed indirectly based on the translational accelerations acquired at different points of the wheel support. The necessary equations follow from rigid body kinematics.

The acceleration $a_{\text {, }}$ of point $P_{i}$ of the rigid body can be obtained from the acceleration $\mathrm{a}_{\mathrm{c}}$ of the center of gravity $\mathrm{C}$ according to

$a_{i}=a_{c}+\dot{\omega} \times r_{G}+\omega \times\left(\omega \times r_{G}\right) \quad, \quad i=1(1) n$,

with the relative vectors $r_{C}$ from $C$ to the $n$ measuring points $P$, and the vector of angular velocities $\omega$.

If the goal is to validate a rigid body model for small oscillations of the wheel support, described by a cartesian frame at the center of gravity, then the quadratic term $\omega \times\left(\omega \times r_{\mathrm{G}}\right)$ of Eq. (1) vanishes. Now, to compute $\dot{\omega}$ the difference vectors $\Delta \mathbf{a}_{p}=\mathbf{a}_{\mathrm{i}}-\mathrm{a}_{\text {, }}$ are introduced leading for $\mathrm{n}>1$ to an overdetermined set of linear equations,

$\left[\begin{array}{c}\tilde{\mathbf{r}}_{21} \\ \overline{\mathbf{r}}_{31} \\ \vdots \\ \tilde{\mathbf{r}}_{\mathrm{a}(\mathrm{a}-1)}\end{array}\right] \dot{\omega}=\left[\begin{array}{c}\Delta \mathbf{a}_{12} \\ \Delta \mathbf{a}_{13} \\ \vdots \\ \Delta \mathbf{a}_{(\mathrm{a}-1) \mathrm{w}}\end{array}\right]$,

where the skewsymmetric tensors $\tilde{\mathbf{r}}_{\mathrm{j}}$ of the corresponding difference vectors $r_{j i}=r_{j}-r$ represent the vector products $r_{j} \times \dot{\omega}$. The regularity of the coefficient matrix in Eq. (2) depends on the position of the measuring points at the rigid body.

Finally, the translational acceleration $\mathrm{a}_{\mathrm{c}}$ of the center of gravity can be calculated from one of the $n$ relations given with Eq. (1). Under practical conditions the best results were obtained by averaging more than one relation. The twist serves as a basis to animate the real motion or to calculate the actual axes of rotation at certain time steps during one vibration period. The time history of location and direction of the rotation axis is an efficient tool to classify the vibration behaviour of the rigid body under consideration.

The instantaneous rotation axis $d(t)$ at the time $t$ is given by

$d(t)=r_{D}(t)+\lambda \omega(t)$,

where the axis point $D$ is defined by normal projection of position vector $r_{C}$ to the rotation axis. The rotation axis is parallel to the vector of angular velocity $\omega$. The unit of scalar $\lambda$ is $[\mathrm{m} \cdot \mathrm{s}]$. The position vector $\mathbf{r}_{\mathrm{D}}$ is given by 
$\mathbf{r}_{\mathrm{D}}=\mathbf{r}_{\mathrm{C}}+\frac{1}{\omega^{\top} \boldsymbol{\omega}}\left(\mathbf{v}_{\mathrm{c}} \times \omega\right)$

For the example presented in this paper seven translational acceleration signals at four different measuring points were taken into account, see Fig. 4. Thus, in Eq. (2) one has to delete all rows containing not measured accelerations.

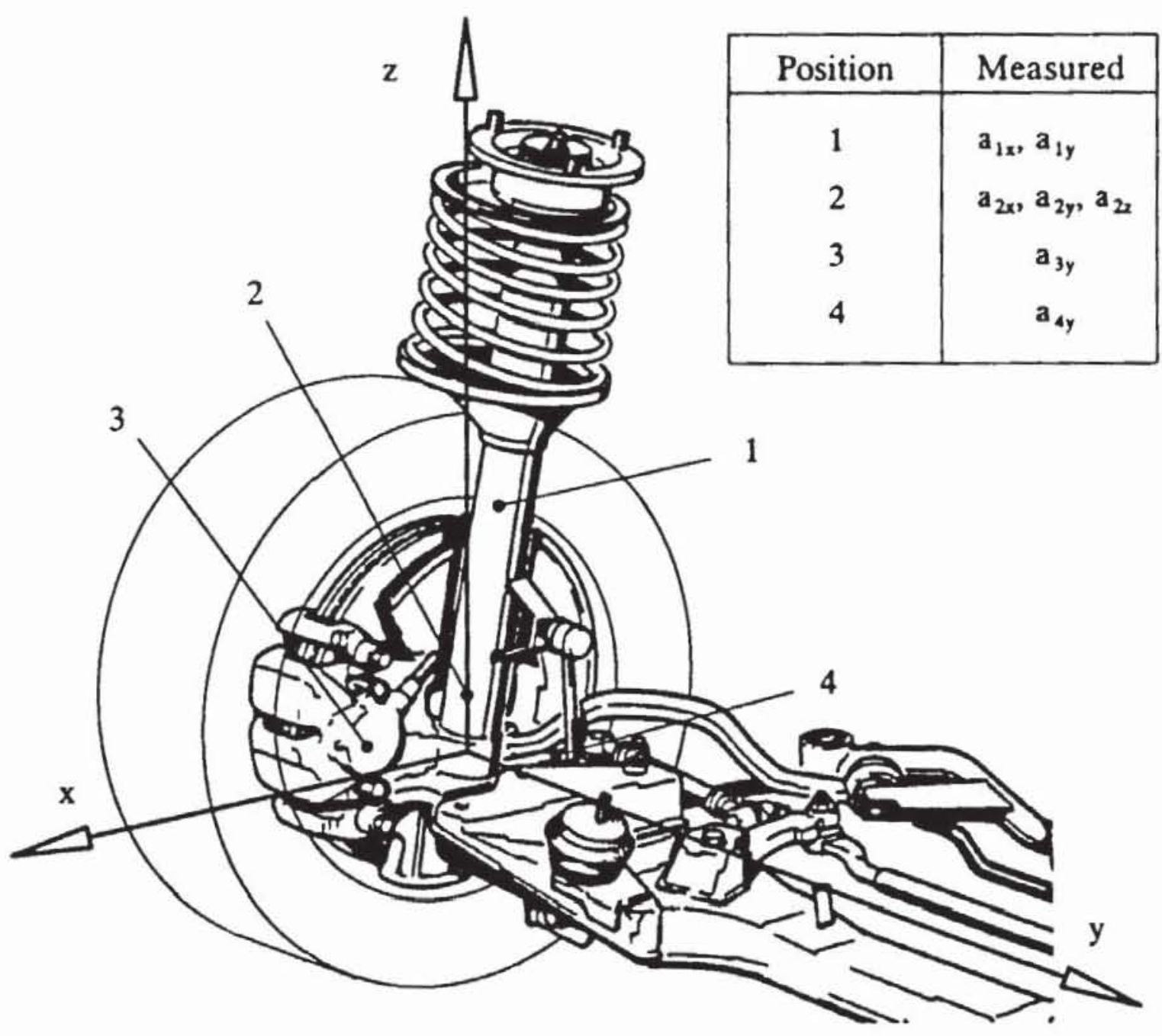

Fig. 4: Measuring points at the right front wheel support

From the instantaneous rotation axis for several different stationary vibration frequencies, one recognizes changing vibration behaviour. Therefore for two frequencies Fig. 5 and 6 show the cartesian coordinates of point D during one vibration period and a 3-dimensional representation of the corresponding directions at discrete time steps. At $16 \mathrm{~Hz}$, where the wheel support is in a resonance, the axes remain almost parallel and the coordinates of $D$ are constant if the singular122 
ity at $|\omega|=0$ is omitted, see Fig. 5. Thus, the wheel support undertakes an almost plain rotation around a fixed spatial axis at $16 \mathrm{~Hz}$. The singularity is due to the equal phase angles of the components of $\omega$ in the case of a plane motion.

At the frequency of $19 \mathrm{~Hz}$ the vibration behaviour is completely different. Now, there is a general spatial motion of the wheel support. During one vibration period the instantaneous rotation axes are neither fixed nor parallel, see Fig. 6.
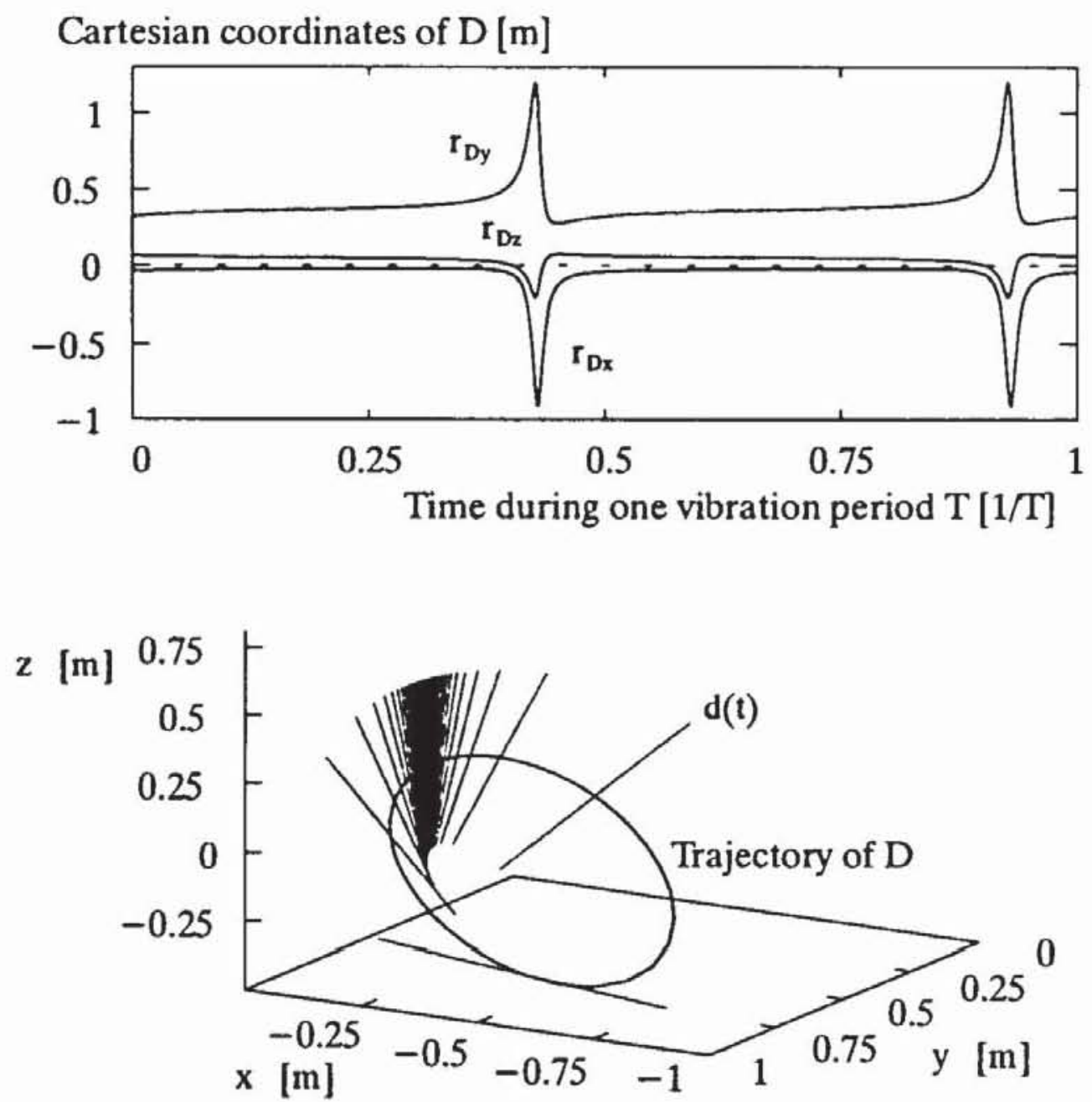

Fig. 5: Spatial motion of the wheel support at $16 \mathrm{~Hz}$ 

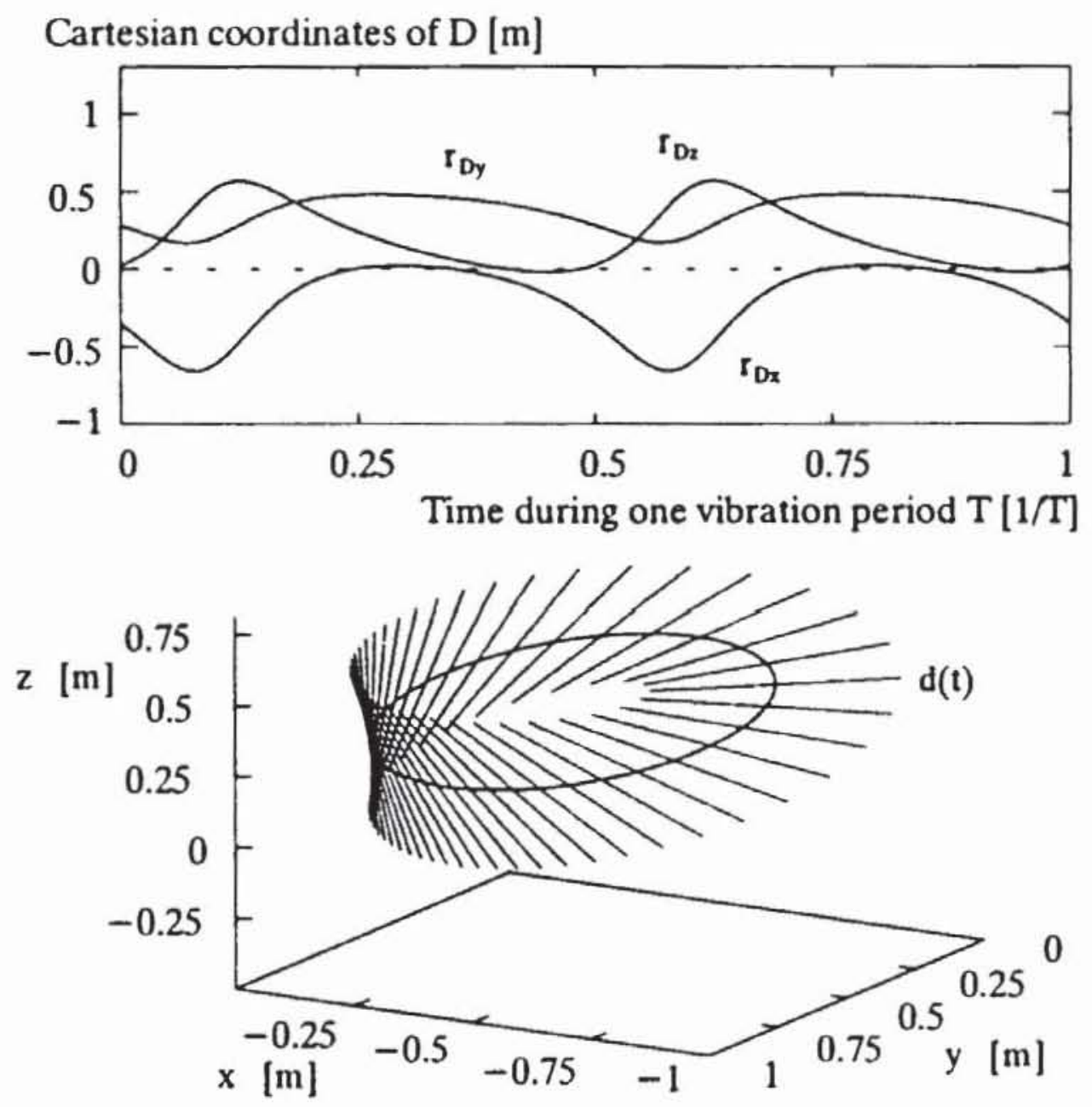

Fig. 6: Spatial motion of the wheel support at $19 \mathrm{~Hz}$

\section{PARAMETER IDENTIFICATION}

The second example deals with parameter identification. Once the validation of the mechanical model is achieved, the multibody system model might be applied for condition monitoring of the vehicle system by parameter identification. The idea is to identify some parameters of the system and of the deterministic excitation, which might be damping coefficients changing due to wear or excentricity and angular location of unbalances, while other parameters, which might be the mass value and moments of inertia of wheel and wheel support are treated as fixed parameters.

The most important information source with this approach is the topology of the mechanical model which has to be determined in advance, e. g. by experiments. Applying the covariance method results in identification relations using that valuable topology information.

The presented method is based on the exact knowledge of any frequency related to the deterministic excitations to be monitored. In the case of unbalancy excitation the devices required for measuring the angular locations of the wheels 124 
are already implemented in modern road vehicles with ABS. However, nonuniformities of rolling wheels result in higher harmonic excitation of the system, too, see e. g. Böhm [1]. Monitoring such complex excitation sources requires an analysis of the complete frequency spectrum of the excitation. An unsymmetric inertia distribution within the tire belt, for instance, results in first harmonic unbalancy excitation as well as in excitation of higher frequencies due to passing the tire-road contact patch.

The accuracy of the presented method is demonstrated on the basis of computer simulated measurements. Then, the mechanical model for system monitoring represents exactly the simulated vehicle. It is assumed that the wheel support is excited by an unbalanced rim and tire stiffness variation due to wheel rotation. The goal is to identify the parameters of the first harmonic excitation based on the knowledge of wheel mass by measuring the acceleration of vehicle body and wheel support as well as the angular location of the wheel. Fig. 7 shows a quarter model of the vehicle. Tire stiffness variation of first order is described by

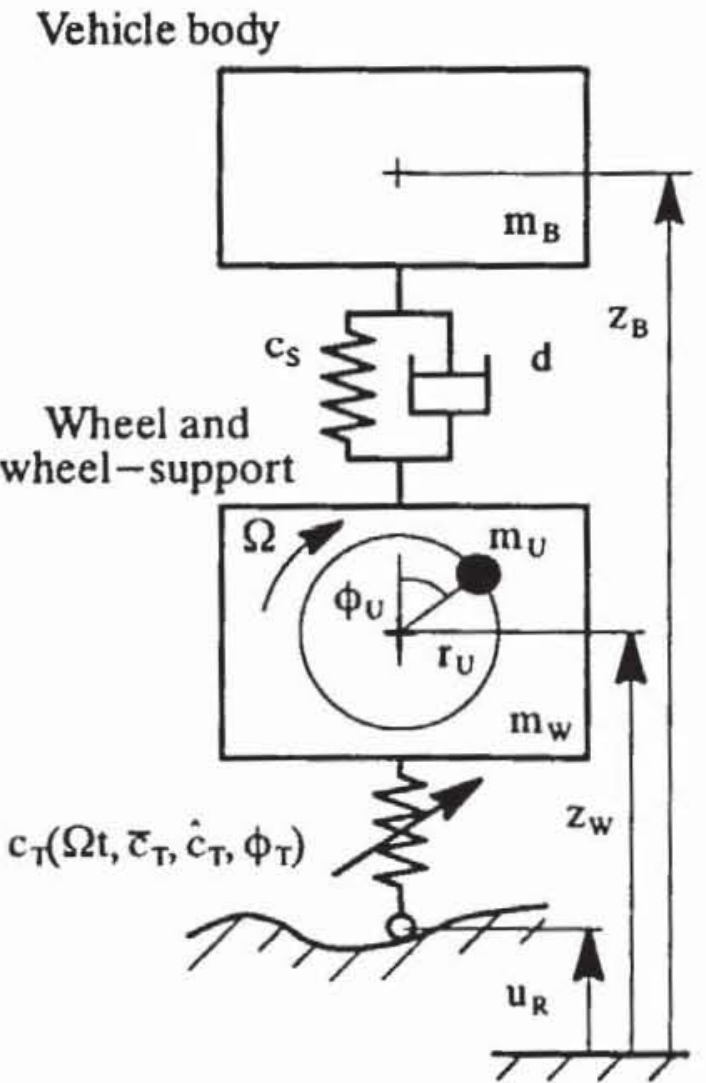

Fig. 7: Parameters of a vehicle model with 2 DOF

$c_{T}(\Omega t)=\tau_{T}+\tilde{c}_{T}(\Omega t)=\tau_{T}+\hat{c}_{T} \cos \left(\Omega t-\phi_{T}\right)$.

Under the assumption of a linear system the equations of motion read as

$\left[\begin{array}{cc}\mathrm{m}_{\mathrm{B}} & 0 \\ 0 & \mathrm{~m}_{\mathrm{w}}\end{array}\right]\left[\begin{array}{l}\ddot{\tilde{z}}_{\mathrm{B}} \\ \ddot{\tilde{z}}_{\mathrm{w}}\end{array}\right]+\left[\begin{array}{cc}\mathrm{d} & -\mathrm{d} \\ -\mathrm{d} & \mathrm{d}\end{array}\right]\left[\begin{array}{l}\dot{\tilde{z}}_{\mathrm{B}} \\ \dot{\tilde{z}}_{\mathrm{w}}\end{array}\right]+\left[\begin{array}{cc}\mathrm{c}_{\mathrm{s}} & -\mathrm{c}_{\mathrm{s}} \\ -\mathrm{c}_{\mathrm{s}} & \mathrm{c}_{\mathrm{s}}+\overline{\mathrm{c}}_{\mathrm{T}}\end{array}\right]\left[\begin{array}{l}\tilde{\mathrm{z}}_{\mathrm{B}} \\ \tilde{\mathrm{z}}_{\mathrm{W}}\end{array}\right]=\left[\begin{array}{l}0 \\ \mathrm{~h}\end{array}\right]$

where the excitation $h=h^{d}+h^{2}$ is given by the deterministic function

$h^{d}=A_{T}\left(\cos \phi_{T}-\cos \left(\Omega t-\phi_{T}\right)\right)+A_{U} \Omega^{2} \cos \left(\Omega t-\phi_{U}\right)$

with

$A_{T}=\frac{\left.\left(m_{B}+m_{W}\right) g \hat{c}_{T}\right)}{\left(\tau_{T}+\hat{c}_{T} \cos \phi_{T}\right)} \quad, \quad A_{U}=m_{U} r_{U}$,

respectively, and a coloured stochastic process $h^{2}$ due to road roughness $u_{R}$, see Popp, Schiehlen [6].

Firstly, the motion is simulated based on Eq. (6) with a given set of parameter values and a coloured noise process corresponding to a smooth road surface. Then, the first harmonics of $z_{B}, z_{W}, \dot{z}_{B}$ and $\dot{z}_{W}$ are extracted by crosscorrelation 
between the measured acceleration signals $\ddot{z}_{B}$ and $z_{w}$ and the model function $\mathrm{m}(\mathrm{t})=\hat{\mathrm{m}} \cos \Omega \mathrm{t}$. Hence, the excitation by the rough road suface is considered as measuring noise and the constant term in Eq. (7) is neglected. Finally, the covariance method is applied to identify the deterministically excited system.

Therefore, the measured signals

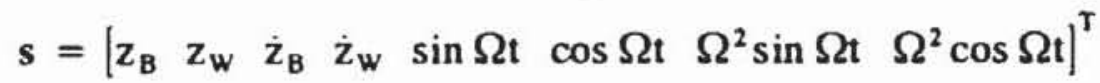

are sent through a user defined linear filter

$\dot{\mathbf{y}}=\mathbf{F y}+\mathbf{V s}$.

Assuming stationarity, $\frac{d}{d t} C_{s y}=0$, one gets the algebraic identification relation

$$
\left(F_{y \dot{z}}+V_{s \dot{z}}\right) M^{\top}-C_{y \dot{z}} D^{T}-C_{y z} K^{T}+C_{y h} \cdot B^{T}=0
$$

with the mass matrix $\mathbf{M}$, the damping matrix $\mathbf{D}$, the stiffness matrix $\mathbf{K}$ defined by Eq. (6) and the input matrix $B$ described by

$$
\mathbf{B h}^{*}=\left[\begin{array}{cccc}
0 & 0 & 0 & 0 \\
A_{T} \cos \phi_{T} & A_{T} \sin \phi_{T} & A_{U} \cos \phi_{U} & A_{U} \sin \phi_{U}
\end{array}\right]\left[\begin{array}{c}
\cos \Omega t \\
\sin \Omega t \\
\Omega^{2} \cos \Omega t \\
\Omega^{2} \sin \Omega t
\end{array}\right]
$$

with respect to the first harmonics of Eq. (7).

In the present case the wheel mass $m_{w}$ is a priori known. Generating Eq. (11) at several different driving speeds and rearranging leads to the inhomogenious overdetermined linear set of equations

$W_{p}=\mathbf{r}$

which might be solved in the sense of least squares to identify the $8 \times 1$-parameter vector $\mathbf{p}$. The row dimension of $\mathbf{W}$ and $\mathbf{r}$ depends on the user defined filter, Eq. (10). The nonlinear parameter combinations occuring in $\mathbf{p}$ due to the parametrization of $\mathbf{B}$ may be solved to get the 8 physical parameters $m_{B}, d, c_{S}, \bar{c}_{T}, \hat{c}_{T}$, $\phi_{\mathrm{T}}, \mathrm{m}_{\mathrm{U}}$ and $\phi_{\mathrm{U}}$.

Finally Fig. 8 shows the absolute errors of the identified angle parameters and the relative errors of the other identified parameters, respectively. These results were obtained using four different driving speeds, each of which with a simulated measuring time of $4 \mathrm{~s}$ and $500 \mathrm{~Hz}$ sampling rate.

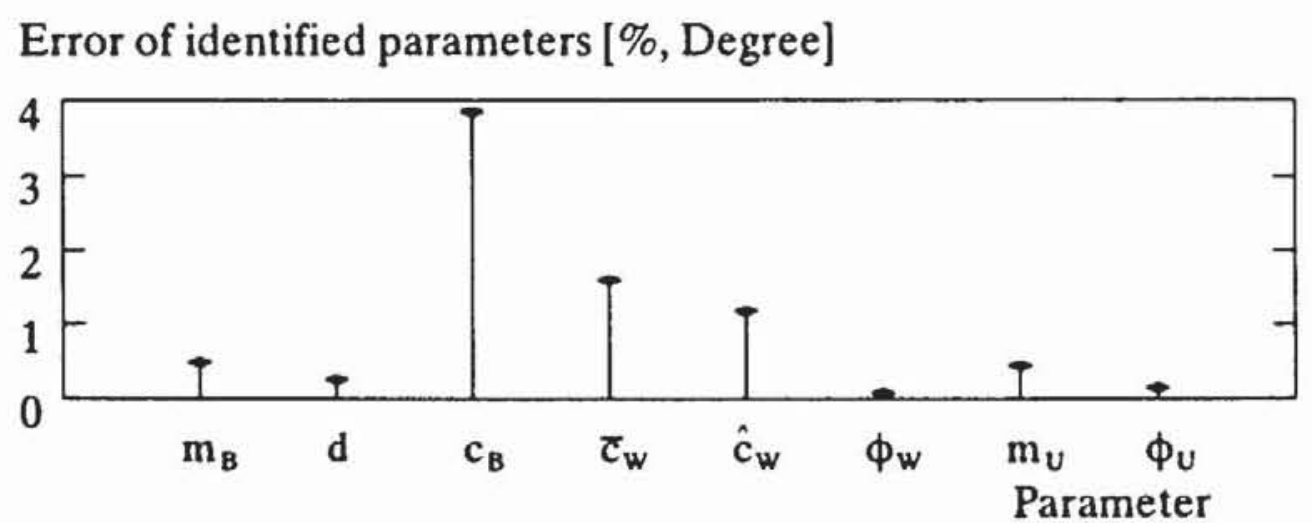

Fig. 8: Result of parameter identification based on a priori known $m_{w}$ and $r_{U}$ 126 


\section{CONCLUSION}

A mobile data acquisition system based on parallel processing using transputer technology was presented. The portable system is qualified to meet a wide variety of applications with dynamic measurements in vehicles combining analog acceleration measurements and digital transducer signals.

Two applications were reported. On the one hand, the vibration monitoring based on signal extraction by crosscorrelation was presented as an appropiate tool for model validation. On the other hand, parameter identification of multi body system models was applied to identify deterministic excitations due to wheel nonuniformities combining measurements while driving with the covariance method.

The results presented show that modern measurement hardware in combination with advanced simulation and parameter identification techniques result in a very efficient tool for vehicle dynamics.

The modular concept of the data acquisition system using transputer technology may be easyly linked to other transputer systems, e. g. to perform real time simulation at the laboratory.

\section{REFERENCES}

1. Böhm, F.; Kollatz, M.: Some Theoretical Models for Computation of Tire Nonuniformities. Fortschr.-Ber. VDI Reihe 12 Nr. 124. Disseldorf: VEI-Verlag, 1989.

2. Hees, G.; ct al.: Measurement in Experimental Cars Using Realtime Computer Systems. SAE 880476. Society of Automotive Engineers, 1989.

3. Eismann, W.: Identifikation von Unwuchtparametern an Fahrzeugrädern während der Fahrt. Ph. D. Thesis. University of Stuttgart, Institute B of Mechanics. To appear.

4. Eismann, W.; Schiehlen, W.: Balancing on the Road. In: Sauvage, G. (ed): The Dynamics of vehicles on roads and on tracks. Proc. 12th IAVSD-Symposium, Lyon, France. Lisse: Swets \& Zeitlinger, 1991, pp. 130-143.

5. Kallenbach, R.: A Covariance Method for Identification of Linear Time-Continuous Systems. In: Barker, H. A., Young, P. C. (ed): Proc. 7th IFAC/IFORS Symposium on Identification and System Parameter Estimation, Vol. 2. Oxford: Pergamon Press, 1985, pp. 1569-1573.

6. Popp, K., Schiehlen, W.: Fahrzeugdynamik. Stuttgart: Teubner, 1993.

7. Zomotor, A.: Fahrwerktechnik: Fahrverhalten. Würzburg: Vogel, 1987. 